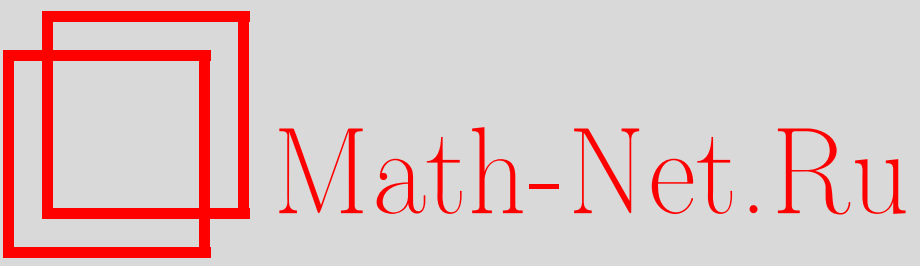

А. М. Зубков, Н. А. Харитонова, Е. В. Хиль, Расстояния между локальными максимумами в последовательностях случайных величин, Теория вероятн. и ее примен., 2011, том 56, выпуск 4, 690-703

DOI: https://doi.org/10.4213/tvp4418

Использование Общероссийского математического портала Math-Net.Ru подразумевает, что вы прочитали и согласны с пользовательским соглашением

http: //www . mathnet.ru/rus/agreement

Параметры загрузки:

IP : 18.208 .226 .222

26 апреля 2023 г., 18:04:19

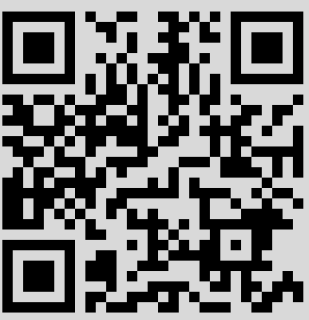


ТЕОРИЯ ВЕРОЯТНОСТЕЙ

Том 56

И ЕЕ ПРИМЕНЕНИЯ

Выпуск 4

2011

(c) 2011 г.

ЗУБКОВ А. М.*, ХАРИТОНОВА Н. А.**, ХИЛЬ Е. В.**

\title{
РАССТОЯНИЯ МЕЖДУ ЛОКАЛЬНЫМИ МАКСИМУМАМИ В ПОСЛЕДОВАТЕЛЬНОСТЯХ СЛУЧАЙНЫХ ВЕЛИЧИН ${ }^{1)}$
}

\begin{abstract}
Найдены явные формулы для распределений расстояний между соседними локальными максимумами в последовательностях случайных величин (как независимых, так и зависимых). Доказана асимптотическая нормальность векторов частот промежутков разных длин между локальными максимумами в отрезке растущей длины. Показано, что распределения промежутков между локальными максимумами зависят от характера зависимости между элементами последовательности.
\end{abstract}

Ключевые слова и фразы: случайные последовательности, локальные максимумы, комбинаторные методы в теории вероятностей.

1. Введение. Пусть $\left\{\xi_{n}, n \in \mathbf{Z}\right\}$ - последовательность действительных случайных величин, $\chi_{n}=I\left\{\xi_{n-1}<\xi_{n}>\xi_{n+1}\right\}(n \in \mathbf{Z})-$ индикаторы локальных максимумов этой последовательности и $\left\{\tau_{j}, j \in \mathbf{Z}\right\}=$ $\left\{n \in \mathbf{Z}: \chi_{n}=1\right\}$ - возрастающая последовательность всех моментов появления локальных максимумов в $\left\{\xi_{n}\right\}$.

$\mathrm{B}$ [1] для независимых случайных величин $\left\{\xi_{n}, n \in \mathbf{Z}\right\}$, имеющих одно и то же непрерывное распределение, изучалось распределение расстояний $\lambda_{j}=\tau_{j+1}-\tau_{j}$ между соседними локальными максимумами: вероятности $\mathbf{P}\left\{\lambda_{j}=k\right\}, k=2,3, \ldots$, представлены в виде многократных интегралов, точные формулы для которых были получены в [7]. Легко видеть, что при этих условиях $\mathbf{P}\left\{\chi_{n}=1\right\}=1 / 3$.

В [1] предлагалось использовать несколько стандартных статистик, основанных на $\lambda_{j}$, для тестирования генераторов случайных чисел. Однако в теоремах о свойствах этих статистик обычно предполагается, что случайные величины $\lambda_{j}$ независимы и одинаково распределены.

\footnotetext{
*Математический институт им. В.А.Стеклова РАН, ул. Губкина, 8, 119991 Москва, Россия; e-mail: zubkov@mi.ras.ru

** Московский государственный университет им. М.В. Ломоносова, механикоматематический факультет, Ленинские горы, 119991 Москва, Россия; e-mail: nexi_weasel@mail.ru, elena_hill@gmail.com

1) Работа выполнена при поддержке РФФИ (грант № 11-01-00139).
} 
В настоящей работе частично описана вероятностная структура последовательности $\left\{\lambda_{j}\right\}$; доказана асимптотическая нормальность векторов, образованных частотами значений $\left\{\lambda_{j}\right\}$ на отрезке последовательности $\left\{\xi_{j}\right\}$.

В качестве примеров рассмотрены частные классы последовательностей зависимых случайных величин, для которых также найдены либо распределения промежутков между локальными максимумами, либо вероятности того, что элемент $\xi_{k}$ последовательности является локальным максимумом.

\section{2. Формулировки результатов.}

Теорема 1. Если случайнье величинь $\left\{\xi_{n}, n \in \mathbf{Z}\right\}$ независимы $и$ имеют одно и то же непрерывное распределение, то

$$
\begin{gathered}
\mathbf{P}\{\lambda=k\}=\frac{3(k+2)(k-1) 2^{k}}{(k+3) !}, \quad k=2,3, \ldots, \\
\mathbf{E} \lambda=3, \quad \mathbf{D} \lambda \approx 1.167, \quad \mathbf{P}\{\lambda>6\} \approx 0.0074 .
\end{gathered}
$$

Кроме того,

$$
\begin{aligned}
\mathbf{P}\left\{\lambda_{1}\right. & \left.=k, \lambda_{2}=l\right\}=\mathbf{P}\left\{\lambda_{1}=l, \lambda_{2}=k\right\} \\
& =\frac{3}{(k+l+3) !} \sum_{m=1}^{k+l+2} \sum_{n=1}^{k+2} C_{m-1}^{n-1} C_{k+l+3-m}^{k+3-n} P(k, n) Q(l, m-n+1),
\end{aligned}
$$

где

$$
\begin{aligned}
& P(k, n)= \begin{cases}k \cdot 2^{k}-(2 k-n+3) \cdot 2^{n-3}, & \text { если } n \geq 3, \\
k \cdot 2^{k}-(k+1), & \text { если } n \in\{1,2\} ;\end{cases} \\
& Q(l, d)= \begin{cases}(2 l-d-1) \cdot 2^{d-3}, & \text { если } d \geq 3, \\
l-1, & \text { если } d \in\{1,2\} .\end{cases}
\end{aligned}
$$

3 а м е ч а н и я. 1. В [1] были получены только формулы (1); приведенное ниже доказательство теоремы 1 (изложенное в дипломной работе Н. А. Харитоновой [2]) использует чисто комбинаторные методы.

2. Доказательство теоремы 1 дословно переносится на случай, когда последовательность $\left\{\xi_{n}, n \in \mathbf{Z}\right\}$ состоит из перестановочных случайных величин, удовлетворяющих условию $\mathbf{P}\left\{\xi_{1}=\xi_{2}\right\}=0$.

Формулы (2) упрощаются, если одно из расстояний равно 2 или 3 :

$$
\begin{aligned}
& \mathbf{P}\left\{\lambda_{1}=k, \lambda_{2}=2\right\}=\frac{\left(k^{2}+9 k+12\right)(k+4)(k-1) \cdot 2^{k}}{(k+5) !}, \\
& \mathbf{P}\left\{\lambda_{1}=k, \lambda_{2}=3\right\}=\frac{(k+2)(k-1) \cdot 2^{k}}{(k+3) !}=\mathbf{P}\left\{\lambda_{1}=k\right\} \mathbf{P}\left\{\lambda_{2}=3\right\} .
\end{aligned}
$$


Таким образом, события $\left\{\lambda_{j}=k\right\}$ и $\left\{\lambda_{j+1}=2\right\}$ зависимы, следовательно, случайные величины последовательности $\left\{\lambda_{j}\right\}_{j \in \mathbf{Z}}$ зависимы; однако событие $\left\{\lambda_{j}=3\right\}$ не зависит от значений $\lambda_{j-1}$ и $\lambda_{j+1}$.

Теорема 2. Собьтия $\left\{\lambda_{j}=3\right\}$ являются регенерируюшими для последовательности $\left\{\lambda_{j}\right\}$, т.е. при условии $\left\{\lambda_{j}=3\right\}$ последовательности $\lambda_{j-1}, \lambda_{j-2}, \ldots$ и $\lambda_{j+1}, \lambda_{j+2}, \ldots$ независимы и одинаково распределень.

Числа появлений локальных максимумов в конечной последовательности $\left\{\xi_{1}, \ldots, \xi_{T}\right\}$ и часто́ты расстояний между ними можно использовать как статистики в критерии согласия с гипотезой $H_{0}$ : элементы последовательности $\left\{\xi_{n}\right\}$ независимь и имеют одно и то же непрерыьное распределение.

Кроме индикаторов локальных максимумов $\chi_{t}=I\left\{\xi_{t-1}<\xi_{t}>\xi_{t+1}\right\}$ введем индикаторы промежутков длины $k$ между соседними локальными максимумами

$$
\chi_{t}^{(k)} \stackrel{\text { def }}{=} I\left\{\chi_{t}=1, \chi_{t+j}=0(j=1, \ldots, k-1), \chi_{t+k}=1\right\} ;
$$

положим

$$
N_{1}(T)=\sum_{i=1}^{T-2} \chi_{i}, \quad N_{k}(T)=\sum_{i=1}^{T-k-2} \chi_{i}^{(k)}, \quad k \geqslant 2 .
$$

Теорема 3. При гипотезе $H_{0}$ распределения векторов $N^{(s)}(T)=$ $\left(N_{1}(T), N_{2}(T), \ldots, N_{s}(T)\right), s \geqslant 2$, при $T \rightarrow \infty$ асимптотически нормальны с параметрами $\left(A^{(s)} T, C_{s} T\right)$, где

$$
A^{(s)}=\left(a_{1}, a_{2}, \ldots, a_{s}\right)=\left(\frac{1}{3}, \frac{1}{3} \mathbf{P}\{\lambda=2\}, \ldots, \frac{1}{3} \mathbf{P}\{\lambda=s\}\right),
$$

а элементы матриць ковариачий $C_{s}=\left\|c_{k m}\right\|_{k, m=1}^{s}$ определяются формулами

$$
\begin{aligned}
c_{11}= & \frac{2}{45}, \\
c_{1 k}=c_{k 1}= & \frac{2}{3} \mathbf{P}\left\{\lambda_{1}=2, \lambda_{2}=k\right\}-\frac{k-1}{9} \mathbf{P}\{\lambda=k\}, \quad k \geqslant 2, \\
c_{k k}= & \frac{1}{3} \mathbf{P}\{\lambda=k\}-\frac{2 k+5}{9} \mathbf{P}^{2}\{\lambda=k\}+\frac{2}{3} \mathbf{P}\left\{\lambda_{1}=k, \lambda_{2}=k\right\} \\
& +\frac{2}{3} \mathbf{P}\left\{\lambda_{1}=k, \lambda_{2}=2, \lambda_{3}=k\right\}, \quad k \geqslant 2, \\
c_{m k}=c_{k m}= & -\frac{k+m+5}{9} \mathbf{P}\{\lambda=k\} \mathbf{P}\{\lambda=m\}+\frac{2}{3} \mathbf{P}\left\{\lambda_{1}=k, \lambda_{2}=m\right\} \\
& +\frac{2}{3} \mathbf{P}\left\{\lambda_{1}=k, \lambda_{2}=2, \lambda_{3}=m\right\}, \quad m>k \geqslant 2 .
\end{aligned}
$$


Формулы для величин $\mathbf{P}\{\lambda=k\}, \mathbf{P}\left\{\lambda_{1}=k, \lambda_{2}=m\right\}$ содержатся в теореме 1 , формула для $\mathbf{P}\left\{\lambda_{1}=k, \lambda_{2}=2, \lambda_{3}=m\right\}$ имеет вид

$$
\begin{aligned}
\mathbf{P}\left\{\lambda_{1}=\right. & \left.k, \lambda_{2}=2, \lambda_{3}=m\right\}=\frac{3 \cdot 2^{k+m}}{(m+1) !(k+1) !(k+m+3)(k+m+5)} \\
\times( & \frac{k m(k+m+5)^{2}-(k+m+3)\left((k+m)^{2}+6(k+m)-3\right)}{(k+3)(m+3)} \\
& \left.+\frac{2(k+m+1)}{C_{k+m+2}^{m+1}}\right) ;
\end{aligned}
$$

ее доказательство не приводится ввиду громоздкости. Точные формулы для первых моментов величины, тесно связанной с $N_{1}(T)$ (максимальной длины альтернирующей подпоследовательности в случайной равновероятной перестановке) были получены в [8].

Как известно, если случайный вектор $Z=\left(z_{1}, \ldots, z_{s}\right)$ имеет $s$ мерное нормальное распределение с вектором средних $a$ и невырожденной ковариационной матрицей $C$, то случайный вектор $C^{-1 / 2}(Z-a)$ имеет $s$-мерное нормальное распределение с параметрами $(0, E)$, где $E-$ единичная $(s \times s)$-матрица, поэтому случайная величина

$$
\left\|C^{-1 / 2}(Z-a)\right\|^{2}=\left(C^{-1 / 2}(Z-a), C^{-1 / 2}(Z-a)\right)=\left(C^{-1}(Z-a), Z-a\right)
$$

имеет распределение хи-квадрат с $s$ степенями свободы. Отсюда и из теоремы 3 следует, что при $T \rightarrow \infty$ распределение случайной величины

$$
\frac{1}{T}\left(C_{s}^{-1}\left(N^{(s)}(T)-A^{(s)} T\right), N^{(s)}(T)-A^{(s)} T\right)
$$

сходится к распределению хи-квадрат с $s$ степенями свободы; это позволяет стандартным образом строить простые критерии согласия с гипотезой $H_{0}$.

Приведем примеры, показывающие, что при некоторых альтернативных гипотезах распределения векторов $N^{(s)}(T)$ при $T \rightarrow \infty$ имеют асимптотики, отличные от указанных в теореме 3.

Пусть случайные величины $\left\{\xi_{n}, n \in \mathbf{Z}\right\}$ независимы и имеют одно и то же непрерывное распределение, а $\xi_{n}^{*}=\xi_{n}+\xi_{n+1}, n \in \mathbf{Z}$. Пусть $\chi_{n}^{*}=$ $I\left\{\xi_{n-1}^{*}<\xi_{n}^{*}>\xi_{n+1}^{*}\right\}(n \in \mathbf{Z})$ - индикаторы локальных максимумов в последовательности $\left\{\xi_{n}^{*}\right\}_{n \in \mathbf{Z}}$ и $\lambda^{*}$ - длина промежутка между соседними локальными максимумами последовательности $\left\{\xi_{n}^{*}\right\}$, а

$$
\chi_{t}^{*(k)} \stackrel{\text { def }}{=} I\left\{\chi_{t}^{*}=1, \chi_{t+j}^{*}=0(j=1, \ldots, k-1), \chi_{t+k}^{*}=1\right\}
$$

- индикаторы промежутков длины $k$ между соседними локальными максимумами. Положим

$$
N_{1}^{*}(T)=\sum_{i=1}^{T-2} \chi_{i}^{*}, \quad N_{k}^{*}(T)=\sum_{i=1}^{T-k-2} \chi_{i}^{*(k)}, \quad k \geqslant 2 .
$$


Теорема 4. Справедливы соотношения

$$
\begin{aligned}
& \mathbf{P}\left\{\chi_{n}^{*}=1\right\}=\frac{1}{4}, \quad n \in \mathbf{Z}, \\
& \mathbf{P}\left\{\lambda^{*}=2 k\right\}=\frac{4}{[(k+2) !]^{2}}\left(k^{2}-\frac{2}{k+2}\right) C_{2 k+1}^{k}, \\
& \mathbf{P}\left\{\lambda^{*}=2 k+1\right\}=\frac{8 k(k+1)}{(k+2) !(k+3) !} C_{2 k+1}^{k}, \quad k=1,2, \ldots, \\
& \mathbf{E} \lambda^{*}=4, \quad \mathbf{D} \lambda^{*} \approx 2.117, \quad \mathbf{P}\left\{\lambda^{*}>8\right\} \approx 0.0081 .
\end{aligned}
$$

Следствие. Для последовательности $\xi_{n}^{*}=\xi_{n}+\xi_{n+1}, n \in \mathbf{Z}$, nостроенной по независимым случайньлм величинам $\xi_{n}, n \in \mathbf{Z}, c$ одними тем же непрерьвньм распределением, распределения векторов $\left(N_{1}^{*}(T), N_{2}^{*}(T), \ldots, N_{s}^{*}(T)\right), s \geqslant 2$, при $T \rightarrow \infty$ асимптотически нормальнь с вектором математических ожиданий

$$
\left(\frac{1}{4}, \frac{1}{4} \mathbf{P}\left\{\lambda^{*}=2\right\}, \ldots, \frac{1}{4} \mathbf{P}\left\{\lambda^{*}=s\right\}\right) .
$$

Утверждение 1. Если $\xi_{n}, n \in \mathbf{Z},-$ последовательность независимых случайных величин, имеющих стандартное нормальное распределение, а $\widetilde{\xi}_{n}=\xi_{n}+c \xi_{n+1}, n \in \mathbf{Z}, c=$ const, $m o$

$$
\mathbf{P}\left\{\widetilde{\xi}_{n-1}<\widetilde{\xi}_{n}>\widetilde{\xi}_{n+1}\right\}=\frac{1}{4}+\frac{1}{2 \pi} \arcsin \frac{(c-1)^{2}}{2\left(c^{2}-c+1\right)} .
$$

Утверждение 2. Если $\xi_{n}, n \in \mathbf{Z},-$ последовательность независимых случайных величин, имеюших равномерное распределение на отрезке $[0,1], a \xi_{n}^{\prime}=\xi_{n}+c \xi_{n+1}, n \in \mathbf{Z}, c=$ const, $m o$

$$
\mathbf{P}\left\{\xi_{n-1}^{\prime}<\xi_{n}^{\prime}>\xi_{n+1}^{\prime}\right\}= \begin{cases}\frac{1}{3}-\frac{c}{12\left(c^{2}-c+1\right)}, & c<0, \\ \frac{1}{3}-\frac{c+c^{2}-c^{3}}{12}, & 0 \leq c<1, \\ \frac{1}{3}-\frac{c^{2}+c-1}{12 c^{3}}, & c \geq 1 .\end{cases}
$$

Теорема 4 и утверждения 1, 2 показывают, что описанные выше статистические критерии будут отличать гипотезу $H_{0}$ от ряда альтернатив.

3. Доказательства теорем. Хорошо известно, что если случайные величины $\xi_{1}, \ldots, \xi_{n}$ независимы и имеют одно и то же непрерывное распределение, то перестановка $\sigma=\sigma\left(\xi_{1}, \ldots, \xi_{n}\right)=\left(\sigma_{1}, \ldots, \sigma_{n}\right)$ чисел $1, \ldots, n$, в которой $\sigma_{k}$ при каждом $k$ равно порядковому номеру $\xi_{k}$ в вариационном ряду $\xi_{(1)}<\xi_{(2)}<\cdots<\xi_{(n)}$ (т. е. $\left.\sigma_{k}=\left|\left\{j \in\{1, \ldots, n\}: \xi_{j} \leqslant \xi_{k}\right\}\right|\right)$, 
имеет равномерное распределение на множестве всех $n$ ! перестановок порядка $n$.

Тогда $\left\{\xi_{i}<\xi_{j}\right\}=\left\{\sigma_{i}<\sigma_{j}\right\}$ при любых $i, j \in\{1, \ldots, n\}$. Поэтому можно сводить вычисление вероятностей событий, определяемых неравенствами между величинами $\xi_{1}, \ldots, \xi_{n}$, к подсчету количества перестановок, удовлетворяющих таким же условиям.

Д о к а з а т е л ь с т в о т е о р е м ы 1. Найдем сначала вероятности $\mathbf{P}\left\{\lambda_{j}=k\right\}$. Так как последовательность $\left\{\xi_{i}\right\}$ (и, значит, $\left\{\lambda_{j}\right\}$ ) однородна по времени, то можно считать, что $j=0$ и $\tau_{0}=2$. Пользуясь тем, что $\left\{\chi_{t}=0\right\}=\left\{\xi_{t}<\max \left\{\xi_{t-1}, \xi_{t+1}\right\}\right\}$, находим:

$$
\begin{aligned}
\mathbf{P} & \{\lambda=k\}=\frac{\mathbf{P}\left\{\chi_{2}=1, \chi_{3}=\cdots=\chi_{k+1}=0, \chi_{k+2}=1\right\}}{\mathbf{P}\left\{\chi_{2}=1\right\}} \\
& =\frac{\mathbf{P}\left\{\xi_{1}<\xi_{2}>\xi_{3}, \xi_{k+1}<\xi_{k+2}>\xi_{k+3}, \xi_{i}<\max \left(\xi_{i-1}, \xi_{i+1}\right), 3 \leqslant i \leqslant k+1\right\}}{\mathbf{P}\left\{\xi_{1}<\xi_{2}>\xi_{3}\right\}} \\
& =\frac{Q(k+3)}{(k+3) !(1 / 3)}=\frac{Q(k+3) \cdot 3}{(k+3) !}
\end{aligned}
$$

где $Q(k+3)$ - количество перестановок порядка $k+3$, обладающих ровно двумя локальными максимумами: в точках 2 и $k+2$.

Найдем $Q(k+3)$. Заметим, что число $k+3$ - глобальный максимум - может находиться только в одном из двух локальных максимумов, причем эти варианты симметричны. Следовательно, $Q(k+3)$ в 2 раза больше числа таких перестановок с $\sigma_{2}=k+3, \sigma_{k+1}<\sigma_{k+2}>\sigma_{k+3}$, что между этими двумя локальными максимумами элементы $\sigma_{j}$ перестановки сначала строго убывают, потом строго возрастают, образуя «овраг».

Лемма 1. Количество перестановок порядка $n$, последовательность элементов которых образует сначала один убьвающий участок, а затем один возрастающий участок (возможно, один из них пуст), равно $2^{\text {n-1 }}$.

Д о к а з а т е л ь с т в о. Действительно, в такой перестановке элемент 1 всегда располагается на стыке двух участков, и перестановка полностью задается выбором чисел для участка убывания, т.е. выбором некоторого подмножества из $\{2, \ldots, n\}$. Таким образом искомое число перестановок равно количеству подмножеств $(n-1)$-элементного множества, т.е. $2^{n-1}$.

Вернемся к доказательству теоремы 1 и рассмотрим возможные значения $\sigma_{k+2}$.

1. $\sigma_{k+2}=k+2$. В этом случае перестановка определяется выбором значений $\sigma_{1}, \sigma_{k+3}$, а оставшиеся числа должны образовать «овраг». Получаем $(k+1) k \cdot 2^{k-2}$ перестановок. 
2. $\sigma_{k+2}=t, 3<t<k+2$. Здесь для значения $\sigma_{k+3}$ возможны $t-1$ вариантов. Далее, если $\sigma_{1} \in\{1, \ldots, t-1\} \backslash\left\{\sigma_{k+3}\right\}(t-2$ вариантов), то $\sigma_{3}=k+2>\sigma_{4}=k+1>\cdots>\sigma_{k-t+4}=t+1$, а оставшиеся $t-3$ чисел образуют «овраг», что в итоге дает $(t-1)(t-2) \cdot 2^{t-4}$ перестановок. Если же $\sigma_{1} \in\{t+1, \ldots, k+2\}\left(k-t+2\right.$ вариантов), то $\sigma_{3}>\cdots>$ $\sigma_{k-t+3}$ и $\left\{\sigma_{3}, \ldots, \sigma_{k-t+3}\right\}=\{t+1, \ldots, k+2\} \backslash\left\{\sigma_{1}\right\}$, а оставшиеся числа, меньшие $t$ ( $t-2$ чисел), образуют «овраг», что дает еще $(t-1)(k-t+$ $2) \cdot 2^{t-3}$ перестановок. Значит, всего в рассматриваемом случае имеется $(t-1)(2 k-t+2) \cdot 2^{t-4}$ перестановок. (Заметим, что эта формула дает правильный результат и при $t=k+2$.)

3. $\sigma_{k+2}=3$. В этом случае для $\sigma_{k+3}$ возможны 2 варианта, для $\sigma_{1}$ возможны $(k-1)$ вариантов, а остальные числа выстраиваются в цепочку по убыванию. Получаем $2(k-1)$ перестановок, что совпадает со значением $(t-1)(2 k-t+2) \cdot 2^{t-4}-1$ при $t=3$.

Таким образом,

$$
\begin{aligned}
Q(k+3) & =2\left(\sum_{t=3}^{k+2}(t-1)(2 k-t+2) \cdot 2^{t-4}-1\right) \\
& =\sum_{t=3}^{k+2}(t-1)(2 k-t+2) \cdot 2^{t-3}-2 .
\end{aligned}
$$

Докажем по индукции, что

$$
Q(k+3)=(k+2)(k-1) \cdot 2^{k} .
$$

Действительно, при $k=2$ получаем $Q(5)=16=(2+2)(2-1) \cdot 2^{2}$. Далее,

$$
\begin{aligned}
Q(k+4)-Q(k+3) & =\sum_{t=3}^{k+2}(t-1) 2^{t-2}+(k+2)(k+1) \cdot 2^{k} \\
& =\left.\frac{d}{d s} \frac{s^{k+2}-s^{2}}{s-1}\right|_{s=2}+(k+2)(k+1) \cdot 2^{k}=\left(k^{2}+5 k+2\right) \cdot 2^{k},
\end{aligned}
$$

что совпадает с разностью правых частей (3):

$(k+3) k \cdot 2^{k+1}-(k+2)(k-1) \cdot 2^{k}=\left(2 k^{2}+6 k-\left(k^{2}+k-2\right)\right) \cdot 2^{k}=\left(k^{2}+5 k+2\right) \cdot 2^{k}$.

Тем самым формула (3) доказана.

Следовательно,

$$
\mathbf{P}\{\lambda=k\}=\frac{Q(k+3) \cdot 3}{(k+3) !}=\frac{3(k+2)(k-1) \cdot 2^{k}}{(k+3) !} .
$$

Математическое ожидание и дисперсия $\lambda$ вычисляются непосредственно:

$$
\begin{gathered}
\mathbf{E} \lambda=\sum_{k=2}^{\infty} k \frac{3(k+2)(k-1) \cdot 2^{k}}{(k+3) !}=3 \\
\mathbf{D} \lambda=\sum_{k=2}^{\infty}(k-3)^{2} \frac{3(k+2)(k-1) \cdot 2^{k}}{(k+3) !}=3\left(e^{2}-7\right) \approx 1.167
\end{gathered}
$$


Вероятности $\mathbf{P}\left\{\lambda_{1}=k, \lambda_{2}=l\right\}$, т.е. совместное распределение двух соседних расстояний, тоже находятся перечислением перестановок, в которых $\sigma_{2}, \sigma_{k+2}, \sigma_{l+k+2}$ и только они являются локальными максимумами. Формула (2) фактически представляет собой формулу полной вероятности по значениям $\sigma_{k+3}=m, m=1, \ldots, k+l+2$, и по рангу $n$ (порядковому номеру в вариационном ряду) этого значения среди $\sigma_{1}, \ldots, \sigma_{k+3}$.

Найдем количество подходящих перестановок при фиксированных $m$ и $n$ : для значений $\sigma_{1}, \ldots, \sigma_{k+2}$ нужно выбрать $n-1$ чисел из $m-1$ чисел, меньших $m$, и $k+3-n$ чисел из $k+l+3-m$ чисел, больших $m$. Такой выбор можно осуществить $C_{m-1}^{n-1} \cdot C_{k+l+3-m}^{k+3-n}$ способами. Далее, необходимо, чтобы в последовательности $\sigma_{1}, \ldots, \sigma_{k+2}, \sigma_{k+3}=m$ значения $\sigma_{2}$ и $\sigma_{k+2}$ были локальными максимумами с «оврагом» между ними. Количество таких перестановок порядка $k+3$ обозначим $P(k, n)$. Кроме того, последовательность $\sigma_{k+3}=m, \sigma_{k+4}, \ldots, \sigma_{k+l+3}$ должна содержать единственный локальный максимум $\sigma_{k+l+2}$. Количество таких перестановок порядка $l+1$ обозначим $Q(l, m-n+1)$, где $m-n+1$ - порядковый номер числа $m$ среди значений $\sigma_{k+3}, \ldots, \sigma_{k+l+3}$.

Вычисление множителей $P(k, n)$ и $Q(l, m-n+1)$ производится аналогично доказательству первой части теоремы.

Чтобы вычислить $P(k, n)$, необходимо найти число таких перестановок порядка $k+3$, что $\sigma_{k+3}=n$, элементы $\sigma_{2}$ и $\sigma_{k+2}-$ локальные максимумы, и других локальных максимумов нет. Рассмотрим возможные значения $\sigma_{k+2}$ при $3 \leqslant n \leqslant k+1$.

1. $\sigma_{k+2}=k+3$. Число таких перестановок равно $Q(k+3) /(2(k+$ $2))=(k-1) \cdot 2^{k-1}$. Деление на 2 возникает из-за того, что фиксировано место расположения глобального максимума, деление на $k+2$ - так как фиксировано одно из $k+2$ возможных значений $\sigma_{k+3}$.

2. $\sigma_{k+2}=k+2$. Тогда $\sigma_{2}=k+3$, и число подходящих перестановок равно $k \cdot 2^{k-2}$, где $k$ - количество возможных значений $\sigma_{1}$ (отличных от $\left.k+2, k+3, \sigma_{k+3}=n\right)$, а $2^{k-2}-$ число способов образования «оврага» между $\sigma_{2}$ и $\sigma_{k+2}$.

3. $\sigma_{k+2}=t, n+1 \leqslant t \leqslant k+1$. Тогда $\sigma_{2}=k+3$, и число подходящих перестановок определяется так же, как в случае 2 вычисления $Q(k)$, с учетом того, что значение $\sigma_{k+3}$ фиксировано. Таким образом, получается $(t-2) \cdot 2^{t-4}+(k-t+2) \cdot 2^{t-3}=(2 k-t+2) \cdot 2^{t-4}$ перестановок.

Итак, при $3 \leqslant n \leqslant k+1$

$$
\begin{aligned}
P(k, n) & =(k-1) \cdot 2^{k-1}+k \cdot 2^{k-2}+\sum_{t=n+1}^{k+1}(2 k-t+2) \cdot 2^{t-4} \\
& =k \cdot 2^{k}-(2 k-n+3) \cdot 2^{n-3} .
\end{aligned}
$$

Если $n=k+2$, то возможен только случай 1 , и $P(k, k+2)=(k-$ $1) \cdot 2^{k-1}$, что соответствует полученной ранее формуле. 
Если $n=2$, то справедливы те же рассуждения, но в случае 3 при $t=3$ не будет первого слагаемого. Поэтому

$$
P(k, 2)=k \cdot 2^{k}-(2 k-n+3) \cdot 2^{n-3}-(n-1) \cdot 2^{n-3}=k \cdot 2^{k}-(k+1) .
$$

Если $n=1$, то случай 3 при $t=2$ невозможен, а при $t=3$ сумма не включает первое слагаемое. Получаем

$$
\begin{aligned}
P(k, 1) & =(k-1) \cdot 2^{k-1}+\sum_{t=4}^{k+2}(t-2) \cdot 2^{t-4}+\sum_{t=3}^{k+2}(k-t+2) \cdot 2^{t-3} \\
& =k \cdot 2^{k}-(k+1) .
\end{aligned}
$$

Чтобы вычислить $Q(l, d)$, нужно найти число перестановок чисел $1, \ldots, l+1$ с $\sigma_{1}=d$, имеющих единственный локальный максимум $\sigma_{l}\left(\sigma_{1}\right.$ не считается локальным максимумом, даже если $\left.\sigma_{1}>\sigma_{2}\right)$.

Рассмотрим возможные значения $\sigma_{1}$.

1. $\sigma_{1}=d=l+1$. В этом случае $\sigma_{l} \in\{3, \ldots, l\}$. Если $\sigma_{l}=u$, то $\sigma_{l+1}$ может принимать любое из $u-1$ значений, меньших $u$, и «овраг» между 1-м и $l$-м элементами перестановки полностью определяется выбором подмножества значений, образующих монотонно возрастающий участок от $\min _{2 \leqslant r<l} \sigma_{r}$ до $\sigma_{l}$. Количество таких подмножеств равно $2^{u-3}$, значит, при $d=l+1$

$$
Q(l, d)=\sum_{u=3}^{l}(u-1) \cdot 2^{u-3}=(l-2) \cdot 2^{l-2}=(2 l-d-1) \cdot 2^{d-3} .
$$

2. $\sigma_{1}=d \in\{3, \ldots, l\}$. Тогда $\sigma_{l}=l+1, \sigma_{l+1}=u \in\{1, \ldots, l\} \backslash\{d\}$ и перестановка однозначно определяется выбором подмножества значений, образующих монотонно убывающий участок от $\sigma_{1}$ до $\min _{2 \leqslant r<l} \sigma_{r}$. Количество таких подмножеств равно $2^{d-2}$ при $u>d$ и $2^{d-3}$ при $u<d$. Значит, при $d \in\{3, \ldots, l\}$

$$
Q(l, d)=\sum_{u=d+1}^{l} 2^{d-2}+\sum_{u=1}^{d-1} 2^{d-3}=2^{d-3}(2 l-d-1) .
$$

3. $\sigma_{1}=d \in\{1,2\}$. В этих случаях $\sigma_{l}=l+1$ и перестановка однозначно определяется выбором значения $\sigma_{l+1} \in\{1, \ldots, l\} \backslash\left\{\sigma_{1}\right\}$, поэтому

$$
Q(l, d)=l-1 .
$$

Теорема 1 доказана.

Доказ ательство те оремы 2. Так как $\left\{\lambda_{j}=3\right\}=$ $\left\{\exists t: \tau_{j}=t-1, \tau_{j+1}=t+2\right\}$ и $\left\{\tau_{j}=t-1, \tau_{j+1}=t+2\right\}=\left\{\xi_{t-2}<\right.$ $\left.\xi_{t-1}>\xi_{t}, \xi_{t+1}<\xi_{t+2}>\xi_{t+3}\right\}$, а последовательность $\left\{\xi_{n}\right\}$ стационарна в 
узком смысле, то теорема 2 следует из более общего утверждения: $n p u$ условии $\left\{\xi_{t-2}<\xi_{t-1}>\xi_{t}, \xi_{t+1}<\xi_{t+2}>\xi_{t+3}\right\}$ любие собьтия $A_{-} \in \mathscr{F}_{-\infty}^{t}$, $A_{+} \in \mathscr{F}_{t+1}^{\infty}$ независимь (здесь $\mathscr{F}_{a}^{b}$ обозначает $\sigma$-алгебру событий, порожденную случайными величинами $\left\{\xi_{t}\right\}_{t=a}^{b}$ ).

Действительно, события $\left\{\xi_{t-2}<\xi_{t-1}>\xi_{t}\right\}$ и $\left\{\xi_{t+1}<\xi_{t+2}>\xi_{t+3}\right\}$ независимы, события $A_{-} \cap\left\{\xi_{t-2}<\xi_{t-1}>\xi_{t}\right\} \in \mathscr{F}_{-\infty}^{t}$ и $A_{+} \cap\left\{\xi_{t+1}<\right.$ $\left.\xi_{t+2}>\xi_{t+3}\right\} \in \mathscr{F}_{t+1}^{\infty}$ тоже независимы. Поэтому

$$
\begin{aligned}
\mathbf{P}\left\{A_{-}\right. & \left.\cap A_{+} \mid \exists j: \tau_{j}=t-1, \tau_{j+1}=t+2\right\} \\
= & \mathbf{P}\left\{A_{-} \cap A_{+} \mid\left\{\xi_{t-2}<\xi_{t-1}>\xi_{t}\right\} \cap\left\{\xi_{t+1}<\xi_{t+2}>\xi_{t+3}\right\}\right\} \\
= & \frac{\mathbf{P}\left\{A_{-} \cap\left\{\xi_{t-2}<\xi_{t-1}>\xi_{t}\right\} \cap A_{+} \cap\left\{\xi_{t+1}<\xi_{t+2}>\xi_{t+3}\right\}\right\}}{\mathbf{P}\left\{\left\{\xi_{t-2}<\xi_{t-1}>\xi_{t}\right\} \cap\left\{\xi_{t+1}<\xi_{t+2}>\xi_{t+3}\right\}\right\}} \\
& =\frac{\mathbf{P}\left\{A_{-} \cap\left\{\xi_{t-2}<\xi_{t-1}>\xi_{t}\right\}\right\} \mathbf{P}\left\{A_{+} \cap\left\{\xi_{t+1}<\xi_{t+2}>\xi_{t+3}\right\}\right\}}{\mathbf{P}\left\{\xi_{t-2}<\xi_{t-1}>\xi_{t}\right\} \mathbf{P}\left\{\xi_{t+1}<\xi_{t+2}>\xi_{t+3}\right\}} \\
& =\mathbf{P}\left\{A_{-} \mid \xi_{t-2}<\xi_{t-1}>\xi_{t}\right\} \cdot \mathbf{P}\left\{A_{+} \mid \xi_{t+1}<\xi_{t+2}>\xi_{t+3}\right\} \\
& =\mathbf{P}\left\{A_{-} \mid \tau_{j}=t-1, \tau_{j+1}=t+2\right\} \cdot \mathbf{P}\left\{A_{+} \mid \tau_{j}=t-1, \tau_{j+1}=t+2\right\} .
\end{aligned}
$$

Доказ а те ль с тв о т е о ре мы 3 . Асимптотическая нормальность распределений векторов $\left(N_{1}(T), N_{2}(T), \ldots, N_{s}(T)\right), s \geqslant 2$, при $T \rightarrow \infty$ следует из того, что случайные величины $N_{k}(T)$ являются суммами конечно-зависимых индикаторов, а для сумм конечно-зависимых ограниченных величин центральная предельная теорема доказана, например, в [3], [6]. Перенос этой теоремы на векторный случай обосновывается теоремой Крамера-Вольда [4].

Асимптотики математических ожиданий и ковариаций аппроксимирующих многомерных нормальных распределений совпадают с асимптотиками математических ожиданий и ковариаций случайных величин $N_{k}(T)$.

Из определения случайных величин $N_{k}(T)$ следует, что

$$
\mathbf{E} N_{1}(T)=(T-2) \mathbf{P}\left\{\chi_{1}=1\right\}=\frac{T-2}{3},
$$

$$
\mathbf{E} N_{k}(T)=(T-k-2) \mathbf{P}\left\{\chi_{1}^{(k)}=1\right\}=\frac{T-k-2}{3} \mathbf{P}\{\lambda=k\}, \quad k \geqslant 2 .
$$

Выведем формулы для вторых моментов. Пользуясь соотношениями

$$
\operatorname{Cov}\left(\chi_{i}, \chi_{j}\right)=\left\{\begin{array}{l}
\mathbf{D} \chi_{i}=\frac{2}{9}, \quad i=j, \\
-\left(\mathbf{P}\left\{\chi_{i}=1\right\}\right)^{2}=-\frac{1}{9}, \quad|i-j|=1, \\
\mathbf{P}\left\{\chi_{i}=1\right\} \mathbf{P}\{\lambda=2\}-\mathbf{P}^{2}\left\{\chi_{i}=1\right\}=\frac{1}{45}, \quad|i-j|=2, \\
0, \quad|i-j| \geqslant 3,
\end{array}\right.
$$


находим:

$$
\mathbf{D} N_{1}(T)=\mathbf{D} \sum_{i=1}^{T-2} \chi_{i}=\sum_{i=1}^{T-2} \sum_{j=1}^{T-2} \operatorname{Cov}\left(\chi_{i}, \chi_{j}\right)=\frac{2 T+2}{45} .
$$

Далее, так как при $k \geqslant 2$

$$
\begin{aligned}
& \operatorname{Cov}\left(\chi_{i}, \chi_{j}^{(k)}\right) \\
& =\left\{\begin{array}{l}
\frac{1}{3} \mathbf{P}\{\lambda=k\}-\frac{1}{3} \frac{1}{3} \mathbf{P}\{\lambda=k\}=\frac{2}{9} \mathbf{P}\{\lambda=k\}, \quad i \in\{j, j+k\}, \\
-\frac{1}{3} \frac{1}{3} \mathbf{P}\{\lambda=k\}, \quad i \in\{j-1, \ldots, j+k+1\} \backslash\{j, j+k\}, \\
\frac{1}{3} \mathbf{P}\left\{\lambda_{1}=2, \lambda_{2}=k\right\}-\frac{1}{9} \mathbf{P}\{\lambda=k\}, \quad i \in\{j-2, j+k+2\}, \\
0, \quad i \leqslant j-3, i \geqslant j+k+3,
\end{array}\right.
\end{aligned}
$$

то

$$
\begin{aligned}
& \operatorname{Cov}\left(N_{1}(T), N_{k}(T)\right)=\sum_{i=1}^{T-2} \sum_{j=1}^{T-k-2} \operatorname{Cov}\left(\chi_{i}, \chi_{j}^{(k)}\right) \\
& =\frac{2(T-k-4)}{3} \mathbf{P}\left\{\lambda_{1}=2, \lambda_{2}=k\right\}-\frac{T(k-1)-k^{2}+13 k-4}{9} \mathbf{P}\{\lambda=k\} \\
& =(1+o(1)) T\left(\frac{2}{3} \mathbf{P}\left\{\lambda_{1}=2, \lambda_{2}=k\right\}-\frac{k-1}{9} \mathbf{P}\{\lambda=k\}\right), \quad T \rightarrow \infty .
\end{aligned}
$$

Аналогично, при $k \geqslant 2$

$$
\begin{aligned}
& \operatorname{Cov}\left(\chi_{i}^{(k)}, \chi_{j}^{(k)}\right) \\
& =\left\{\begin{array}{l}
\frac{1}{3} \mathbf{P}\{\lambda=k\}\left(1-\frac{1}{3} \mathbf{P}\{\lambda=k\}\right), \quad i=j, \\
\frac{1}{3} \mathbf{P}\left\{\lambda_{1}=k, \lambda_{2}=k\right\}-\frac{1}{9}(\mathbf{P}\{\lambda=k\})^{2}, \quad|i-j|=k, \\
-\frac{1}{9}(\mathbf{P}\{\lambda=k\})^{2}, \quad|i-j| \leqslant k+1, i \neq j,|i-j| \neq k, \\
\frac{1}{3} \mathbf{P}\left\{\lambda_{1}=k, \lambda_{2}=2, \lambda_{3}=k\right\}-\frac{1}{9}(\mathbf{P}\{\lambda=k\})^{2}, \quad|i-j|=k+2, \\
0, \quad|i-j| \geqslant k+3,
\end{array}\right.
\end{aligned}
$$

и поэтому при $k \geqslant 2$

$$
\begin{aligned}
\mathbf{D} N_{k}(T) & =\mathbf{D} \sum_{i=1}^{T-k-2} \chi_{i}^{(k)}=\sum_{i=1}^{T-k-2} \sum_{j=1}^{T-k-2} \operatorname{Cov}\left(\chi_{i}^{(k)}, \chi_{j}^{(k)}\right) \\
& =\frac{T-k-2}{3} \mathbf{P}\{\lambda=k\}-\frac{T(2 k+5)-(k+2)(3 k+8)}{9} \mathbf{P}^{2}\{\lambda=k\}
\end{aligned}
$$




$$
\begin{aligned}
& +\frac{2(T-2 k-2)}{3} \mathbf{P}\left\{\lambda_{1}=k, \lambda_{2}=k\right\} \\
& +\frac{2(T-2 k-4)}{3} \mathbf{P}\left\{\lambda_{1}=k, \lambda_{2}=2, \lambda_{3}=k\right\} \\
= & \frac{T(1+o(1))}{3}\left(\mathbf{P}\{\lambda=k\}-\frac{2 k+5}{3} \mathbf{P}^{2}\{\lambda=k\}\right. \\
& \left.\quad+2 \mathbf{P}\left\{\lambda_{1}=k, \lambda_{2}=k\right\}+2 \mathbf{P}\left\{\lambda_{1}=k, \lambda_{2}=2, \lambda_{3}=k\right\}\right) .
\end{aligned}
$$

Наконец, при $m>k \geqslant 2, k \neq m$,

$$
\begin{aligned}
& \operatorname{Cov}\left(\chi_{i}^{(k)}, \chi_{j}^{(m)}\right) \\
& =\left\{\begin{aligned}
-\frac{1}{9} \mathbf{P}\{\lambda=k\} \mathbf{P}\{\lambda=m\}, \\
j \in\{i-m-1, \ldots, i+k+1\} \backslash\{i-m, i+k\}, \\
\frac{1}{3} \mathbf{P}\left\{\lambda_{1}=k, \lambda_{2}=m\right\}-\frac{1}{9} \mathbf{P}\{\lambda=k\} \mathbf{P}\{\lambda=m\}, \\
j \in\{i-m, i+k\}, \\
\frac{1}{3} \mathbf{P}\left\{\lambda_{1}=k, \lambda_{2}=2, \lambda_{3}=m\right\}-\frac{1}{9} \mathbf{P}\{\lambda=k\} \mathbf{P}\{\lambda=m\}, \\
j \in\{i-m-2, i+k+2\}, \\
0, \quad j \notin\{i-m-2, \ldots, i+k+2\},
\end{aligned}\right.
\end{aligned}
$$

и поэтому

$$
\begin{aligned}
\operatorname{Cov}( & \left.N_{k}(T), N_{m}(T)\right)=\sum_{i=1}^{T-k-2} \sum_{j=1}^{T-m-2} \operatorname{Cov}\left(\chi_{i}^{(k)}, \chi_{j}^{(m)}\right) \\
= & -\frac{T(k+m+5)-\left(k^{2}+k m+m^{2}+7 k+7 m+16\right)}{9} \mathbf{P}\{\lambda=k\} \mathbf{P}\{\lambda=m\} \\
& +\frac{2(T-k-m-2)}{3} \mathbf{P}\left\{\lambda_{1}=k, \lambda_{2}=m\right\} \\
& +\frac{2(T-k-m-4)}{3} \mathbf{P}\left\{\lambda_{1}=k, \lambda_{2}=2, \lambda_{3}=m\right\} \\
= & \frac{T(1+o(1))}{3}\left(-\frac{k+m+5}{3} \mathbf{P}\{\lambda=k\} \mathbf{P}\{\lambda=m\}\right. \\
& \left.\quad+2 \mathbf{P}\left\{\lambda_{1}=k, \lambda_{2}=m\right\}+2 \mathbf{P}\left\{\lambda_{1}=k, \lambda_{2}=2, \lambda_{3}=m\right\}\right) .
\end{aligned}
$$

Теорема 3 доказана.

Д ок азательст в о т е о ремы 4. Заметим, что

$$
\begin{aligned}
& \mathbf{P}\left\{\xi_{n-1}^{*}<\xi_{n}^{*}>\xi_{n+1}^{*}\right\}=\mathbf{P}\left\{\xi_{n-1}+\xi_{n}<\xi_{n}+\xi_{n+1}>\xi_{n+1}+\xi_{n+2}\right\} \\
& \quad=\mathbf{P}\left\{\xi_{n-1}<\xi_{n+1}, \xi_{n}>\xi_{n+2}\right\}=\mathbf{P}\left\{\xi_{n-1}<\xi_{n+1}\right\} \cdot \mathbf{P}\left\{\xi_{n}>\xi_{n+2}\right\}=\frac{1}{4}
\end{aligned}
$$


Как и при доказательстве теоремы 1 , можно считать первым локальным максимумом $\xi_{2}^{*}$, поскольку последовательность $\left\{\xi_{n}^{*}\right\}$ стационарна в узком смысле. Поэтому

$$
\begin{aligned}
& \mathbf{P}\left\{\lambda^{*}=k\right\}=\mathbf{P}\left\{\xi_{k+1}^{*}<\xi_{k+2}^{*}>\xi_{k+3}^{*}, \xi_{i}^{*}<\max \left\{\xi_{i-1}^{*}, \xi_{i+1}^{*}\right\},\right. \\
& \left.i=3, \ldots, k+1 \mid \xi_{1}^{*}<\xi_{2}^{*}>\xi_{3}^{*}\right\}=\frac{1}{\mathbf{P}\left\{\xi_{1}^{*}<\xi_{2}^{*}>\xi_{3}^{*}\right\}} \sum_{m=3}^{k+1} P(k, m),
\end{aligned}
$$

где

$P(k, m)=\mathbf{P}\left\{\xi_{1}^{*}<\xi_{2}^{*}>\xi_{3}^{*}>\cdots>\xi_{m-1}^{*}>\xi_{m}^{*}<\xi_{m+1}^{*}<\cdots<\xi_{k+2}^{*}>\xi_{k+3}^{*}\right\}$.

Так же, как при вычислении $\mathbf{P}\left\{\xi_{n-1}^{*}<\xi_{n}^{*}>\xi_{n+1}^{*}\right\}$, вероятности $P(k, m)$ представляются в виде произведений вероятностей аналогичных событий, относящихся к величинам $\xi_{n}$ с четными или с нечетными индексами, например,

$$
\begin{aligned}
P(2 k, 2 m)= & \mathbf{P}\left\{\xi_{1}^{*}<\xi_{2}^{*}>\xi_{3}^{*}>\cdots>\xi_{2 m-1}^{*}>\xi_{2 m}^{*}<\cdots<\xi_{2 k+2}^{*}>\xi_{2 k+3}^{*}\right\} \\
= & \mathbf{P}\left\{\xi_{1}<\xi_{3}>\xi_{5}>\cdots>\xi_{2 m+1}<\ldots<\xi_{2 k+3} ;\right. \\
& \left.\xi_{2}>\xi_{4}>\cdots>\xi_{2 m}<\ldots<\xi_{2 k+2}>\xi_{2 k+4}\right\} \\
= & \mathbf{P}\left\{\xi_{1}<\xi_{3}>\xi_{5}>\cdots>\xi_{2 m+1}<\cdots<\xi_{2 k+3}\right\} \\
& \times \mathbf{P}\left\{\xi_{2}>\xi_{4}>\cdots>\xi_{2 m}<\cdots<\xi_{2 k+2}>\xi_{2 k+4}\right\} ;
\end{aligned}
$$

вид такого разложения зависит от четности аргументов функции $P(k, m)$. Вероятности событий в правой части вычисляются с помощью комбинаторных рассуждений, аналогичных проведенным в доказательстве теоремы 1. Ввиду громоздкости промежуточных формул (и того, что последовательность $\left\{\xi_{n}^{*}\right\}$ имеет частный вид) эти вычисления здесь не приводятся.

Д ок а з а т е л ь с т в о с л е д с т в и я. Как и в теореме 3 , асимптотическая нормальность распределений векторов $\left(N_{1}^{*}(T), N_{2}^{*}(T), \ldots\right.$, $\left.N_{s}^{*}(T)\right), s \geqslant 2$, является следствием центральной предельной теоремы для конечно-зависимых случайных величин. Асимптотические формулы для математических ожиданий непосредственно следуют из теоремы 4.

Доказ а тельст т о у т в е р жде н ия 1 . Так как $\widetilde{\xi}_{n}=\xi_{n}+$ $c \xi_{n+1}, n \in \mathbf{Z}, c=$ const, где $\left\{\xi_{n}\right\}$ - последовательность независимых случайных величин, имеющих стандартное нормальное распределение, то

$$
\mathbf{P}\left\{\widetilde{\xi}_{1}<\widetilde{\xi}_{2}>\widetilde{\xi}_{3}\right\}=\mathbf{P}\left\{\xi_{1}+c \xi_{2}<\xi_{2}+c \xi_{3}>\xi_{3}+c \xi_{4}\right\}=\mathbf{P}\left\{\zeta_{1}>0, \zeta_{2}>0\right\},
$$

где $\zeta_{1}=c \xi_{3}-\xi_{1}-(c-1) \xi_{2}, \zeta_{2}=\xi_{2}+(c-1) \xi_{3}-c \xi_{4}$.

Вектор $\left(\zeta_{1}, \zeta_{2}\right)$ имеет двумерное нормальное распределение с нулевым средним и матрицей ковариаций

$$
C=\left(\begin{array}{cc}
2\left(c^{2}-c+1\right) & (c-1)^{2} \\
(c-1)^{2} & 2\left(c^{2}-c+1\right)
\end{array}\right) .
$$


Поэтому (см., например, задачу 3.266 в [5])

$$
\mathbf{P}\left\{\zeta_{1}>0, \zeta_{2}>0\right\}=\frac{1}{4}+\frac{1}{2 \pi} \arcsin \frac{(c-1)^{2}}{2\left(c^{2}-c+1\right)} .
$$

Доказательство утвер ждения 2 . Пусть $P(c)=$ $\mathbf{P}\left\{\xi_{1}^{\prime}<\xi_{2}^{\prime}>\xi_{3}^{\prime}\right\}$. Покажем, что $P(c)=P(1 / c), c \neq 0$. Действительно, если $c>0$, то

$$
\begin{aligned}
P(c) & =\mathbf{P}\left\{\xi_{1}^{\prime}<\xi_{2}^{\prime}>\xi_{3}^{\prime}\right\}=\mathbf{P}\left\{\xi_{1}+c \xi_{2}<\xi_{2}+c \xi_{3}>\xi_{3}+c \xi_{4}\right\} \\
& =\mathbf{P}\left\{\frac{1}{c} \xi_{1}+\xi_{2}<\frac{1}{c} \xi_{2}+\xi_{3}>\frac{1}{c} \xi_{3}+\xi_{4}\right\}=P\left(\frac{1}{c}\right)
\end{aligned}
$$

а если $c<0$, то

$$
\begin{aligned}
P(c) & =\mathbf{P}\left\{\xi_{1}+c \xi_{2}<\xi_{2}+c \xi_{3}>\xi_{3}+c \xi_{4}\right\} \\
& =\mathbf{P}\left\{1-\xi_{1}+c\left(1-\xi_{2}\right)<1-\xi_{2}+c\left(1-\xi_{3}\right)>1-\xi_{3}+c\left(1-\xi_{4}\right)\right\} \\
& =\mathbf{P}\left\{\xi_{1}+c \xi_{2}>\xi_{2}+c \xi_{3}<\xi_{3}+c \xi_{4}\right\} \\
& =\mathbf{P}\left\{\frac{1}{c} \xi_{1}+\xi_{2}<\frac{1}{c} \xi_{2}+\xi_{3}>\frac{1}{c} \xi_{3}+\xi_{4}\right\}=P\left(\frac{1}{c}\right) .
\end{aligned}
$$

Таким образом, достаточно рассмотреть только случай $|c| \leq 1$. Вычисление вероятностей $P(c)$ сводится к вычислению двукратных интегралов по многоугольным областям; ввиду громоздкости промежуточных формул (и частного вида последовательности) они здесь не приводятся.

\section{СПИСОК ЛИТЕРАТУРЫ}

1. Kuketayev A. Probability distribution of distances between local extrema of random number series. - Вестник Караганд. ун-та, сер. Физика, 2011, т. 62, № 2, с. 21-34; arXiv:math/0611130, 2006.

2. Харитонова Н. А. Распределения расстояний между локальными максимумами случайной последовательности. - Дипл. работа, мех.-мат. ф-т МГУ, 2009, 15 с.

3. Бернштейн C.H. Распространение предельной теоремы теории вероятностей на суммы зависимых величин. - Успехи матем. наук, 1944, в. 10, с. 65-114.

4. Cramér H., Wold H. Some theorems on distribution functions. - J. London Math. Soc., 1936, v. 11, p. 290-294.

5. Зубков А.М., Севастьянов Б. А., Чистяков В.П. Сборник задач по теории вероятностей. М.: Наука, 1989, 320 с.

6. Ибрагимов И.А., Линник Ю.В. Независимые и стационарно связанные величины. М.: Наука, 1965, 524 с.

7. Oshanin G., Voituriez R., Nechaev S., Vasilyev O., Hivert F. Random patterns generated by random permutations of natural numbers. - Eur. Phys. J. Special Topics, 2007, v. 143, p. 143-157; arXiv:cond-mat/0609718, 2006.

8. Stanley $R$. Longest alternating subsequences of permutations. - Michigan Math. J., 2008 , v. 57 , p. $675-687$. 